\title{
Matrin 3 Is a Component of Neuronal Cytoplasmic Inclusions of Motor Neurons in Sporadic Amyotrophic Lateral Sclerosis
}

\author{
Mikiko Tada, " Hiroshi Doi, * Shigeru Koyano, ${ }^{*}$ Shun Kubota,, Ryoko Fukai, * Shunta Hashiguchi, " Noriko Hayashi, \\ Yuko Kawamoto, ${ }^{*}$ Misako Kunii, ${ }^{*}$ Kenichi Tanaka, ${ }^{*}$ Keita Takahashi, ${ }^{*}$ Yuki Ogawa, ${ }^{*}$ Ryo Iwata, ${ }^{*}$ Shoji Yamanaka, \\ Hideyuki Takeuchi, ${ }^{*}$ and Fumiaki Tanaka*
}

From the Departments of Neurology and Stroke Medicine* and Pathology, ${ }^{\dagger}$ Yokohama City University Graduate School of Medicine, Yokohama, Japan

\author{
Accepted for publication \\ October 19, 2017 \\ Address correspondence to \\ Fumiaki Tanaka, M.D., Ph.D., \\ Department of Neurology and \\ Stroke Medicine, Yokohama \\ City University Graduate \\ School of Medicine, 3-9 \\ Fukuura, Kanazawa-ku, Yoko- \\ hama 236-0004, Japan. E-mail: \\ ftanaka@yokohama-cu.ac.jp.
}

\begin{abstract}
Mutations in the MATR3 gene have been identified as a cause of familial amyotrophic lateral sclerosis, but involvement of the matrin 3 (MATR3) protein in sporadic amyotrophic lateral sclerosis (SALS) pathology has not been fully assessed. We immunohistochemically analyzed MATR3 pathology in the spinal cords of SALS and control autopsy specimens. MATR3 immunostaining of the motor neuron nuclei revealed two distinct patterns: mild and strong staining. There were no differences in the ratio of mild versus strong nuclear staining between the SALS and control cases. MATR3-containing neuronal cytoplasmic inclusions (NCIs) were observed in $60 \%$ of SALS cases. Most motor neurons with MATR3-positive NCIs exhibited a mild nuclear staining pattern. Although $16.8 \%$ of NCIs positive for transactivating response region DNA-binding protein 43 (TDP-43) were estimated as double-labeled by MATR3, no MATR3-positive or TDP-43-negative NCIs were observed. Although a previous study found that MATR3positive NCIs are present only in cases with C9orf72 hexanucleotide repeat expansion, ubiquitinpositive granular NCIs were not observed in the cerebellum, which have been reported as specific to C9orf72-related ALS. Six ALS cases were confirmed to be negative for the GGGGCC hexanucleotide. Our results reveal that MATR3 is a component of TDP-43-positive NCIs in motor neurons, even in SALS, and indicate the broader involvement of MATR3 in ALS pathology and the heterogeneity of TDP-43-positive NCIs. (Am J Pathol 2018, 188: 507-514; https://doi.org/10.1016/j.ajpath.2017.10.007)
\end{abstract}

Amyotrophic lateral sclerosis (ALS) is a neurodegenerative disorder characterized clinically by progressive weakness of skeletal muscles and neuropathologically by degeneration of upper and lower motor neurons. Ubiquitinated inclusions in the cytoplasm of motor neurons are the pathologic hallmark of ALS and the related disorder frontotemporal lobar degeneration (FTLD). Transactivating response region DNA-binding protein 43 (TDP-43) is a major component of these inclusions in most ALS cases. ${ }^{1,2}$ Although mutations in $T A R D B P$, the gene encoding TDP-43, have been identified as a cause of familial ALS (FALS), ${ }^{3,4}$ most sporadic ALS (SALS), FALS, and FTLD cases with TDP-43-positive inclusions are negative for TARDBP mutations. ${ }^{1,2}$ Instead, the C9orf72 mutation is the most common genetic cause of FALS with TDP-43-positive inclusions in white patients. ${ }^{5}$ In addition, fused in sarcoma/translated in liposarcoma (FUS/ TLS) and ubiquilin 2 (UBQLN2) are present in neuronal cytoplasmic inclusions (NCIs) in SALS and FTLD cases without mutations in the encoding genes $F U S^{6,7}$ and UBQLN2, ${ }^{8}$ whereas superoxide dismutase 1 (SOD1) accumulation is restricted to cases with mutations in $S O D 1 .{ }^{9}$ The fact that the products of FALS-related genes are involved in SALS pathology indicates that these proteins play essential roles in the disease progression of FALS and SALS.

Matrin 3 (MATR3) is a $125-\mathrm{kDa}$ inner nuclear matrix protein that binds to DNA and RNA. ${ }^{10,11}$ MATR3 contains

\footnotetext{
Supported by Grant-in-Aid for Young Scientists (B) 15K18367 from the Japan Society for Promotion of the Sciences (M.T.).

Disclosures: None declared.
} 
a nuclear localization signal, ${ }^{12}$ two zinc finger domains predicted to bind DNA, and two RNA recognition motifs. A missense mutation in a domain-less region of MATR 3 causes vocal cord and pharyngeal weakness with distal myopathy. ${ }^{13,14}$ Recently, mutations in MATR3 were identified as a cause of FALS ${ }^{15}$ and were also detected in SALS. ${ }^{16,17}$ In that report, densely stained nuclei of motor neurons and glial cells by MATR3 immunostaining were described as a pathologic finding associated with SALS cases; MATR3positive NCIs were not observed in FALS or SALS cases, except in one patient harboring C9orf72 hexanucleotide repeat expansion. Moreover, MATR3 has not been detected in TDP-43-positive NCIs. ${ }^{15}$ Except for the single report mentioned above, MATR3 pathology in motor neurons has not been investigated in SALS.

Our aim was to elucidate the involvement of MATR3 pathology in SALS. The immunoreactivity of MATR3 was investigated in motor neurons of lumbar spinal cords from 15 autopsied SALS cases, and it was also investigated whether MATR3 co-localizes in TDP-43-positive NCIs. On the basis of our results, we discuss the pathologic role of MATR3 in SALS.

\section{Materials and Methods}

\section{Materials}

The brains and spinal cords of 15 SALS and seven control cases for which autopsies were performed from 2004 to 2015 at the Yokohama City University Hospital were analyzed. This study was approved by the institutional review board of Yokohama City University School of Medicine. The following information from the medical records and clinical summaries was reviewed: age at onset, sex, initial symptoms, and disease duration. It was clinically and neuropathologically confirmed that control cases did not have motor neuron disease. Clinical features of SALS cases (cases 1 to 15) and control cases (cases 16 to 22) are summarized in Table 1.

\section{Repeat-Primed PCR Analysis}

For six cases in which DNA materials were available, GGGGCC hexanucleotide expansion in C9orf72 was screened for using repeat-primed PCR, as reported previously. ${ }^{5}$ Fragment analysis was performed using an ABI PRISM 3500xl (Life Technologies, Carlsbad, CA) and the GeneMapper Software version 3.5 (Applied Biosystems, Foster City, CA). We used samples from patients with the C9orf72 hexanucleotide repeat expansion as controls which were provided from Japanese Consortium for Amyotrophic Lateral Sclerosis Research (Nagoya, Japan).

\section{Characterization of Anti-MATR3 Antibody}

Full-length MATR3 (NM_018834.5) cDNA (pFN21ASDA 0723) was obtained from Kazusa DNA Research Institute
Table 1 Clinical Findings of the Cases Used in This Study

\begin{tabular}{|c|c|c|c|c|c|}
\hline Disease & Case no. & $\begin{array}{l}\text { Age at } \\
\text { death, } \\
\text { years }\end{array}$ & Sex & $\begin{array}{l}\text { Initial symptom } \\
\text { (muscle } \\
\text { weakness) }\end{array}$ & $\begin{array}{l}\text { Disease } \\
\text { duration } \\
\text { months }\end{array}$ \\
\hline \multirow[t]{15}{*}{ ALS } & 1 & 64 & M & Upper limb & 30 \\
\hline & 2 & 54 & $M$ & Upper limb & 24 \\
\hline & 3 & 72 & $M$ & Upper limb & 3 \\
\hline & 4 & 54 & M & Upper limb & 48 \\
\hline & 5 & 87 & $M$ & Upper limb & 29 \\
\hline & 6 & 63 & $M$ & Upper limb & 12 \\
\hline & 7 & 72 & $F$ & Upper limb & 31 \\
\hline & 8 & 64 & M & Upper limb & 23 \\
\hline & 9 & 61 & M & Lower limb & 8 \\
\hline & 10 & 65 & $M$ & Lower limb & 48 \\
\hline & 11 & 79 & M & Lower limb & 75 \\
\hline & 12 & 86 & $M$ & Bulbar & 21 \\
\hline & 13 & 79 & M & Bulbar & 22 \\
\hline & 14 & 69 & $\mathrm{~F}$ & Bulbar & 7 \\
\hline & 15 & 78 & $M$ & Bulbar & 9 \\
\hline Sarcoidosis & 16 & 53 & $M$ & NA & NA \\
\hline DLBCL & 17 & 37 & $M$ & NA & NA \\
\hline CCA & 18 & 89 & $F$ & NA & NA \\
\hline PD & 19 & 71 & M & NA & NA \\
\hline$A D$ & 20 & 84 & $F$ & NA & NA \\
\hline CPA & 21 & 77 & $\mathrm{~F}$ & NA & NA \\
\hline Sarcoidosis & 22 & 80 & $\mathrm{~F}$ & NA & NA \\
\hline
\end{tabular}

$F$, female; $M$, male; $A D$, Alzheimer disease; ALS, amyotrophic lateral sclerosis; CCA, cortical cerebellar atrophy; CPA, cardiac pulmonary arrest; DLBCL, diffuse large B-cell lymphoma; NA, not applicable; PD, Parkinson disease.

(Chiba, Japan). A part of SH-SY5Y or HEK293T cells on 24well plates were transfected with $0.5 \mu \mathrm{g}$ of pFN21ASDA0723 vector using Lipofectamine LTX transfection reagent (Thermo Fisher Scientific, Waltham, MA). The cells with or without transfection were then washed twice with ice-cold phosphatebuffered saline (PBS), fixed for 20 minutes in 4\% paraformaldehyde and PBS, washed twice with PBS, and permeabilized for 5 minutes with $0.1 \%$ Triton X-100 and PBS. Immunostaining was performed using the four anti-MATR3 antibodies listed in Table 2. Goat polyclonal Alexa Fluor 488-conjugated anti-mouse or anti-rabbit IgG (A-11017 or A-11034; Thermo Fisher Scientific) was used as the secondary antibody. Halo-tagged MATR3 was also visualized using the Halo-tag TMR ligand (Promega Corporation, Madison, WI).

For Western blotting, SH-SY5Y or HEK293T cells grown in 10-cm culture dishes were washed twice with ice-cold PBS, lyzed with radioimmunoprecipitation assay buffer [50 mmol/ L Tris hydrochloride ( $\mathrm{pH} 7.5$ ), $150 \mathrm{mmol} / \mathrm{L}$ sodium calcium, $1 \%$ Triton X-100, $0.1 \%$ SDS, $0.5 \%$ sodium deoxycholate, 1 $\mathrm{mmol} / \mathrm{L}$ EDTA, and complete protease inhibitor cocktail (Roche Diagnostics, Mannheim, Germany)], and then briefly sonicated. Cell lysates were subjected to SDS-PAGE followed by Western blotting with four anti-MATR3 antibodies listed in Table 2. Horseradish peroxidase -conjugated sheep polyclonal anti-mouse IgG or donkey polyclonal anti-rabbit IgG (NA931V or NA934V; GE Healthcare UK Ltd., 
Table 2 Antibodies Used for Immunohistochemical Analysis

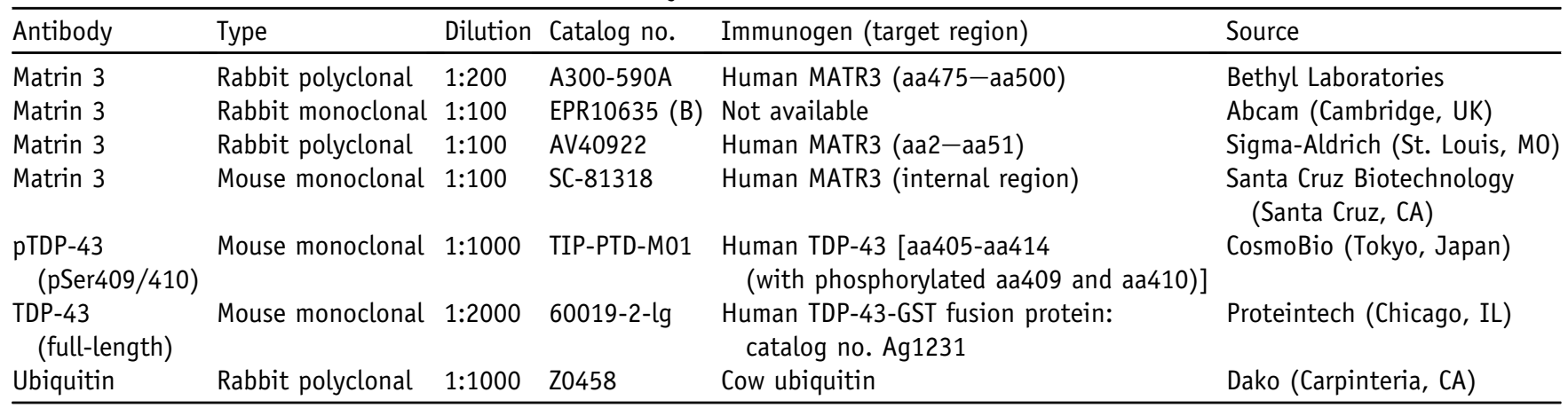

aa, amino acid; MATR3, matrin 3; PTDP-43, phosphorylated transactivating response region DNA-binding protein 43; TDP-43, transactivating response region DNA-binding protein 43.

Buckinghamshire, UK) was used as the secondary antibody. As a control, human MATR3 was prepared as follows. HEK293T cells in 10-cm dishes were transfected with $14 \mu \mathrm{g}$ of vector pFN21ASDA0723. Twenty-four hours after transfection, MATR3 was purified using the Halo-Tag Mammalian Pull-Down system (G6504; Promega Corporation). After elution of MATR3, the Halo-tag was removed by TEV protease (Promega Corporation) cleavage.

\section{Neuropathologic Evaluations}

Each brain and spinal cord was fixed in $20 \%$ neutral buffered formalin (Muto Pure Chemicals, Tokyo, Japan) for 7 to 13 days, embedded in paraffin, and sectioned at a thickness of $6 \mu \mathrm{m}$. Sections from the frontal pole, middle frontal gyrus, motor cortex, temporal pole, superior and the middle temporal gyrus, visual cortex, anterior cingulate gyrus,

Table 3 Pathologic Findings in ALS and Control Cases

\begin{tabular}{|c|c|c|c|c|c|c|c|c|}
\hline \multirow{2}{*}{$\begin{array}{l}\text { Case } \\
\text { no. }\end{array}$} & \multirow{2}{*}{$\begin{array}{l}\text { Brain } \\
\text { weight, g }\end{array}$} & \multirow{2}{*}{$\begin{array}{l}\text { Stage of } \\
\text { TDP }-43^{*}\end{array}$} & \multirow{2}{*}{$\begin{array}{l}\text { Neuronal loss } \\
\text { and gliosis }\end{array}$} & \multicolumn{2}{|c|}{$\begin{array}{l}\text { AHC with NCI/total } \\
\mathrm{AHC}, n(\%)\end{array}$} & \multirow{2}{*}{$\begin{array}{l}\text { Nuclear staining pattern } \\
\text { of cells having } \\
\text { MATR3-positive NCIs }\end{array}$} & \multirow{2}{*}{$\begin{array}{l}\text { Mild staining } \\
\text { nuclei of } \\
\text { AHC, } n(\%)\end{array}$} & \multirow{2}{*}{$\begin{array}{l}\text { Strong staining } \\
\text { nuclei of } \mathrm{AHC} \text {, } \\
n(\%)\end{array}$} \\
\hline & & & & pTDP-43 & MATR3 & & & \\
\hline 1 & 1425 & 4 & 2 & $9 / 37(24)$ & $1 / 45(2)$ & Mild 1 & $28(62)$ & $17(38)$ \\
\hline 2 & 1230 & 1 & 3 & $9 / 40(22)$ & $0 / 43(0)$ & & $20(47)$ & $23(53)$ \\
\hline 3 & 1270 & 4 & 3 & $3 / 23(13)$ & $2 / 18(9)$ & Mild 1, strong 1 & $6(33)$ & $12(67)$ \\
\hline 6 & 1500 & 2 & 1 & $30 / 64(47)$ & $5 / 64(8)$ & Mild 4, strong 1 & $29(45)$ & $35(55)$ \\
\hline 7 & 1450 & 2 & 3 & $18 / 23(78)$ & $0 / 36(0)$ & & $13(36)$ & $23(64)$ \\
\hline 8 & 1330 & 4 & 2 & $5 / 37(14)$ & $1 / 34(3)$ & Mild 1 & $21(43)$ & $28(57)$ \\
\hline 9 & 1320 & 1 & 2 & $16 / 42(38)$ & $1 / 31(3)$ & Mild 1 & $8(26)$ & $23(74)$ \\
\hline 10 & 1340 & 3 & 3 & $19 / 48(40)$ & $2 / 43(4)$ & Strong 2 & $16(37)$ & $27(63)$ \\
\hline 15 & 1160 & 2 & 2 & $9 / 75(12)$ & $3 / 59(5)$ & Mild 3 & $25(42)$ & $34(58)$ \\
\hline \multicolumn{9}{|c|}{ Control cases } \\
\hline 16 & 1500 & & 0 & 0 & 0 & & $28(62)$ & $17(38)$ \\
\hline 17 & 1055 & & 0 & 0 & 0 & & $20(47)$ & $23(53)$ \\
\hline 18 & 1080 & & 0 & 0 & 0 & & $6(33)$ & $12(67)$ \\
\hline 19 & 1390 & & 0 & 0 & 0 & & $1(3)^{\prime}$ & 32 (97) \\
\hline 20 & 1210 & & 0 & 0 & 0 & & $35(64)$ & $20(36)$ \\
\hline 21 & 1150 & & 0 & 0 & 0 & & $29(45)$ & $35(55)$ \\
\hline 22 & 1050 & & 0 & 0 & 0 & & $13(36)$ & $23(64)$ \\
\hline
\end{tabular}

*Stages of pTDP-43 pathology: stage 1, agranular motor cortex, bulbar and spinal somatomotor neurons; stage 2, reticular formation and precerebellar nuclei; stage 3, prefrontal neocortex and basal ganglia; and stage 4, anteromedial areas of the temporal lobe and hippocampal formation (according to the methods described in the article by Brettschneider et $\mathrm{al}^{18}$ ).

${ }^{\dagger}$ Neuronal loss and gliosis of the anterior horn: 0 , none; 1 , mild; 2, moderate; and 3, severe.

AHC, anterior horn cell; NCI, neuronal cytoplasmic inclusion; pTDP-43, phosphorylated transactivating response region DNA-binding protein 43; TDP-43, transactivating response region DNA-binding protein 43. 


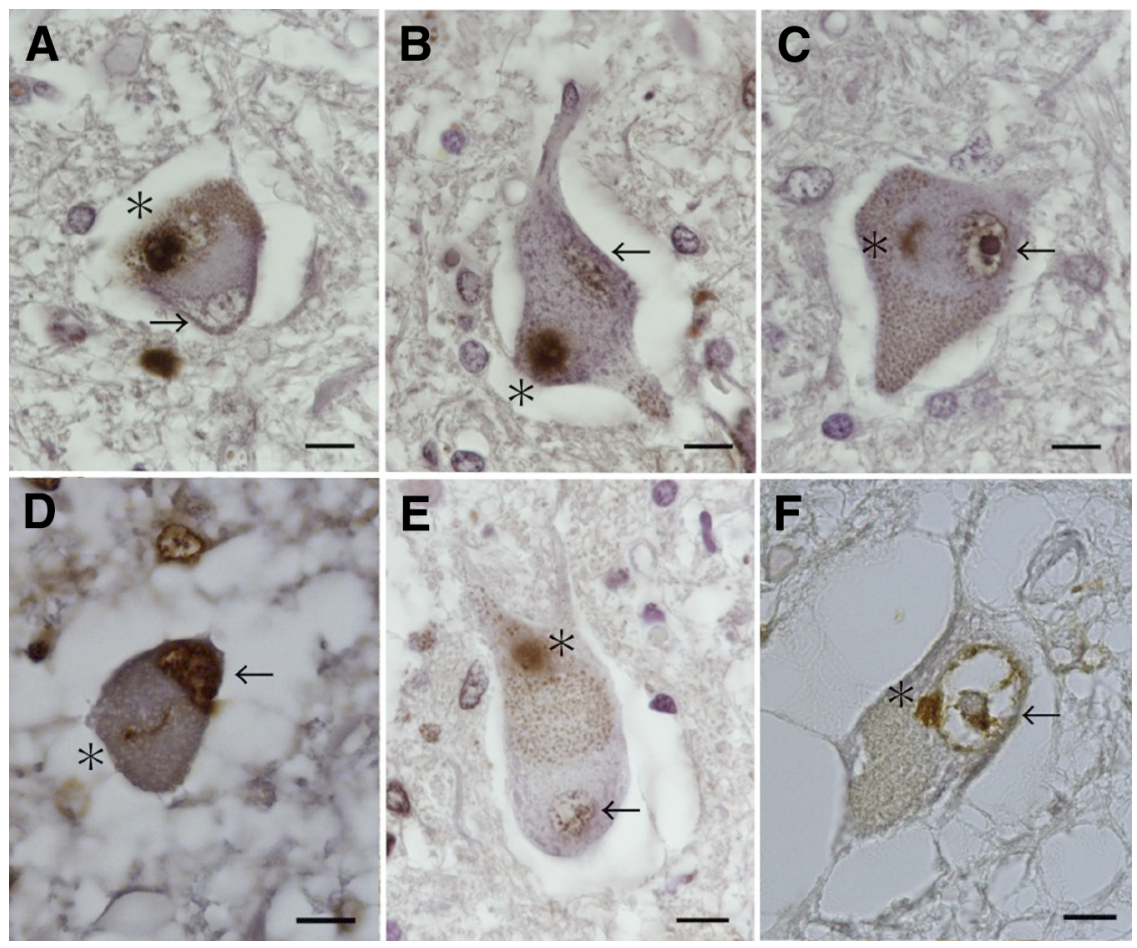

Figure 1 Representative staining pattern of matrin 3 (MATR3)-positive neuronal cytoplasmic inclusions (NCIs) of motor neurons in sporadic amyotrophic lateral sclerosis (SALS) cases. A-D: MATR3-positive NCIs in case 6. A and B: Cytoplasmic round inclusions with mild nuclear staining. C: Cytoplasmic skein-like inclusions with mild nuclear staining. D: Cytoplasmic skein-like inclusions with strong nuclear staining. E: MATR3-positive NCIs in case 8. Cytoplasmic round inclusions with mild nuclear staining. F: MATR3positive NCIs in case 14 . Cytoplasmic round inclusions with mild nuclear staining. Nuclei are indicated by arrows; NCIs, by asterisks. Scale bars $=10 \mu \mathrm{m}$.

hypothalamus, amygdala, hippocampal formation, striatum, globus pallidus, thalamus, midbrain (including the substantial nigra and the red nucleus), upper pons at the level of the locus coeruleus, lower pons at the level of the motor nucleus of the facial nerve, medulla oblongata at the level of the hypoglossal nucleus, cerebellum (including dentate nucleus), cervical spinal cord, thoracic spinal cord, lumbar spinal cord, and sacral spinal cord were stained with hematoxylin and eosin, as well as by the Klüver-Barrera method for conventional neuropathologic studies. For immunohistochemical staining of MATR3 and TDP-43, lumbar spinal cord (at the lumbar enlargement) sections of each case were autoclaved at $121^{\circ} \mathrm{C}$ for 10 minutes, incubated in $100 \%$ formic acid for 5 minutes, and then incubated in $1 \%$ hydrogen peroxide for 20 minutes. Sections were immunostained with the primary antibodies described in Table 2, using the avidin-biotin-peroxidase complex method (ABC Elite; Vector Laboratories, Burlingame, CA). For double staining of MATR3 and TDP-43, Alexa Fluor 568-conjugated anti-mouse IgG antibody (A-11004) and Alexa Fluor 488-conjugated anti-rabbit IgG (A-11008) (Molecular Probes, Eugene, OR) were used as secondary antibodies.

\section{Evaluation of Neurodegeneration and Frequency of NCIs}

The sections of lumbar spinal cord were stained with hematoxylin and eosin to assess the extent and severity of neuronal loss and gliosis, which were graded independently as follows: 0 , none; 1 , mild; 2 , moderate; or 3 , severe.
Immunostained preparations were used to evaluate the subcellular distribution of NCIs that contained TDP-43 or MATR3. To evaluate the frequency of TDP-43-positive and MATR3-positive NCIs of lower motor neurons, the number of anterior horn neurons were counted in two sections from the lumbar spinal cord (L4 or L5).

\section{Statistical Analysis}

Numerical comparisons were performed and assessed with the $U$-test. Differences were considered significant at $P<0.05$.

\section{Results}

All 15 SALS cases were diagnosed with ALS by the conventional neuropathologic evaluation. TDP-43-positive NCIs (skein-like inclusions and round inclusions) were present in the spinal anterior horn cells of all ALS cases (Table $3^{18}$ and Supplemental Figure S1). None of the control cases exhibited TDP-43-positive deposits in brains or spinal cords.

Subcellular localization of MATR3 was evaluated in the spinal cords of SALS and control cases by anti-MATR3 immunostaining. The immunoreactivity of anti-MATR3 antibodies was examined in SH-SY5Y and HEK293T cells. It was confirmed that all four anti-MATR3 antibodies reacted with MATR3 in both cell lines and were suitable for use in Western blotting and immunohistochemistry (Supplemental Figures S2 and S3). However, specificity of AV40922 was lower than that of the other antibodies. 


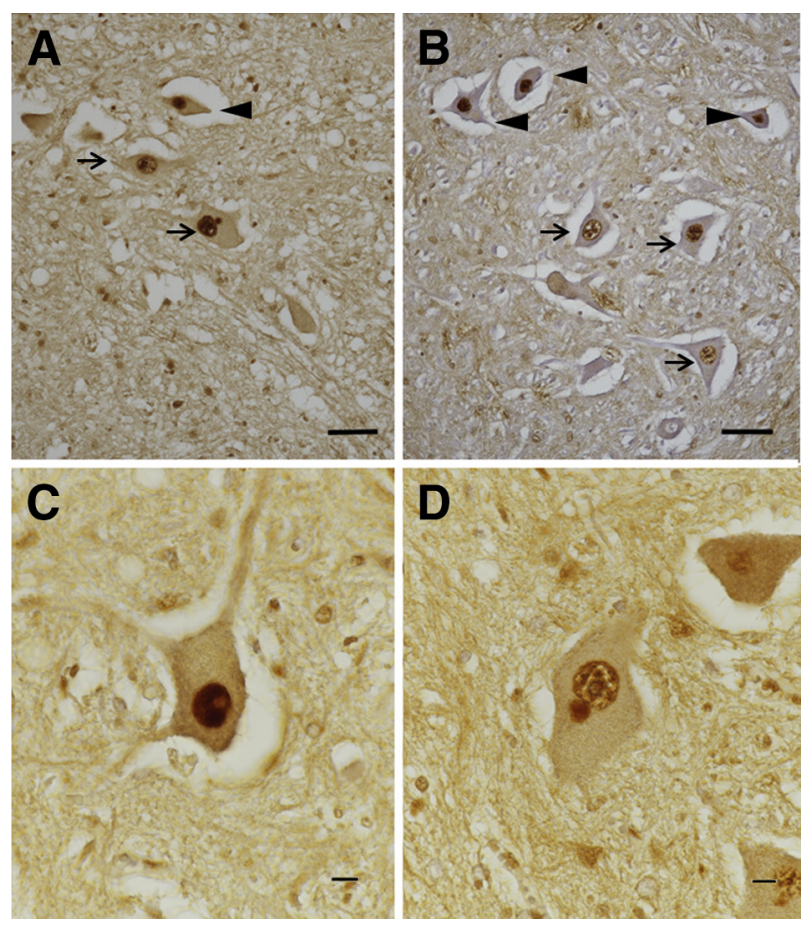

Figure 2 Matrin 3 (MATR3) staining pattern of lumbar spinal cords in sporadic amyotrophic lateral sclerosis (SALS) and control cases. A: Immunohistochemical staining with anti-MATR3 antibody in the lumbar cord of SALS case 14. Nuclei of motor neurons exhibit both strong (arrowheads) and mild (arrows) staining patterns. A subset of motor neurons have MATR3-positive neuronal cytoplasmic inclusions (NCIs). B: Immunohistochemical staining with anti-MATR3 antibody in the lumbar cord of control case 17. As in SALS case 14, the nuclei of motor neurons exhibit both strong (arrowheads) and mild (arrows) staining patterns. C: Nucleus of motor neuron showing strong staining pattern (case 15). D: Nucleus of motor neuron showing mild staining pattern with NCI (case 15). Scale bars: $50 \mu \mathrm{m}$ (A and B); $10 \mu \mathrm{m}$ (C and $\mathbf{D})$.

In immunohistochemistry, only a single antibody (A300590A, Bethyl Laboratories, Montgomery, TX) detected MATR3-positive inclusions in the SALS cases tested (Figure 1). Therefore, this antibody was used for further evaluations. MATR3 in the nuclei of motor neurons exhibits two distinct immunostaining patterns: mild and strong (Figure 2). There were no significant differences in the frequency of mild nuclear staining between SALS $(35.0 \% \pm 17.8 \%)$ and control cases $(42.7 \% \pm 22.8 \%)$ (Figure 2 and Table 3). This was also true for the frequency of strong nuclear staining $(64.9 \% \pm 17.8 \%$ for SALS versus $57.2 \% \pm 22.8 \%$ for control). Glial cells also exhibited mild and strong staining patterns in SALS and control cases. These results indicate that the strong nuclear staining of motor neuron and glial cells described in the original report ${ }^{15}$ was not a specific pathologic finding of SALS. Importantly, MATR3-positive NCIs were observed in 2\% to $9 \%$ of motor neurons in nine of 15 SALS cases (Table 3). Although 16 of 18 MATR3-positive NCIs (88.9\%) were round in shape, 2 of $18(11.1 \%)$ were skein-like in shape (Figure 1), similar to those observed in TDP-43 positive NCIs. ${ }^{2}$ Furthermore, most of the motor neurons with MATR3-positive NCIs had a mild nuclear staining pattern (Table 3 and Figure 1).

To confirm whether these SALS cases included C9orf72related ALS, ubiquitin-positive and TDP-43-negative cytoplasmic inclusions were studied in the cerebellum and hippocampus ${ }^{5,19-21}$; however, none of the cases had these pathologic findings. Moreover, six ALS cases [three cases with MATR3-positive NCIs (cases 1, 8, and 14) and three cases without MATR3-positive NCIs (cases 4, 7, and 13)] were negative for the GGGGCC hexanucleotide expansion (Supplemental Figure S4). The MATR3-positive and TDP-43-positive NCIs in the lumbar spinal cord sections from patients with SALS were then separately evaluated. MATR3-positive NCIs were significantly less abundant than TDP-43-positive NCIs $(2.4 \% \pm 2.8 \%$ versus $24.2 \% \pm 19.3 \% ; P<0.0001$ ) (Table 3). Moreover, SALS cases with MATR3-positive NCIs had significantly shorter disease duration than those without MATR3-positive NCIs $(14.2 \pm 10.2$ versus $36.8 \pm 21.2$ months; $P<0.05)$. Other than disease duration, there were no significant differences in clinical characteristics between MATR3-positive and MATR3-negative cases (Table 4).

Finally, the association between MATR3-positive NCIs and TDP-43-positive NCIs was assessed by doublelabeling immunofluorescence staining with anti-TDP-43 and anti-MATR3 antibodies. The co-localization of MATR3

Table 4 Clinical and Pathologic Summary of SALS Cases with and without MATR3-Positive NCIs

\begin{tabular}{llll}
\hline & MATR3-positive NCIs & & \\
\cline { 2 - 4 } Characteristic & Positive cases $(n=9)$ & Negative cases $(n=6)$ & 0.8130 \\
\hline Age at death, years & $70.33 \pm 17.32$ & $70.67 \pm 13.65$ & 0.7974 \\
U/L/B Clinical phenotype, $n$ & $5 / 2 / 2$ & $3 / 1 / 2$ & 0.8662 \\
Sex, male/female & $7 / 2$ & $5 / 1$ & $0.0291^{*}$ \\
Disease duration, months & $14.22 \pm 10.28$ & $36.83 \pm 21.22$ & 0.6070 \\
Brain weight, g & $1328 \pm 94.80$ & $1325 \pm 180.19$ & 0.5932 \\
Stage $1 / 2 / 3 / 4$ of pTDP-43, $n$ & $2 / 2 / 1 / 4$ & $1 / 1 / 0 / 4$ & \\
\hline
\end{tabular}

Data are expressed as means \pm SD unless indicated otherwise.

${ }^{*} P<0.05$.

MATR3, matrin 3; SALS, sporadic amyotrophic lateral sclerosis; U/L/B, upper limb/lower limb/bulbar. 
MATR3
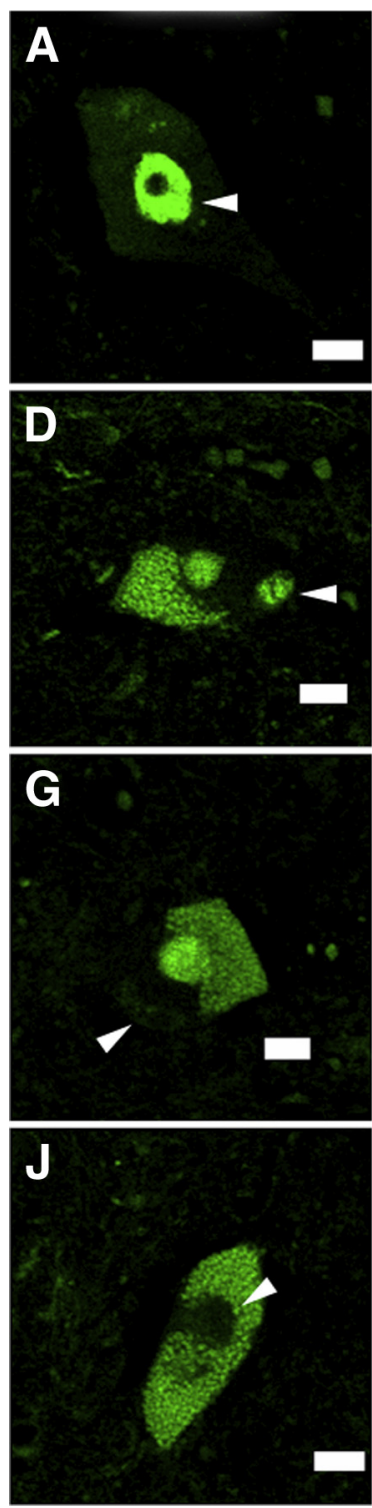

TDP-43
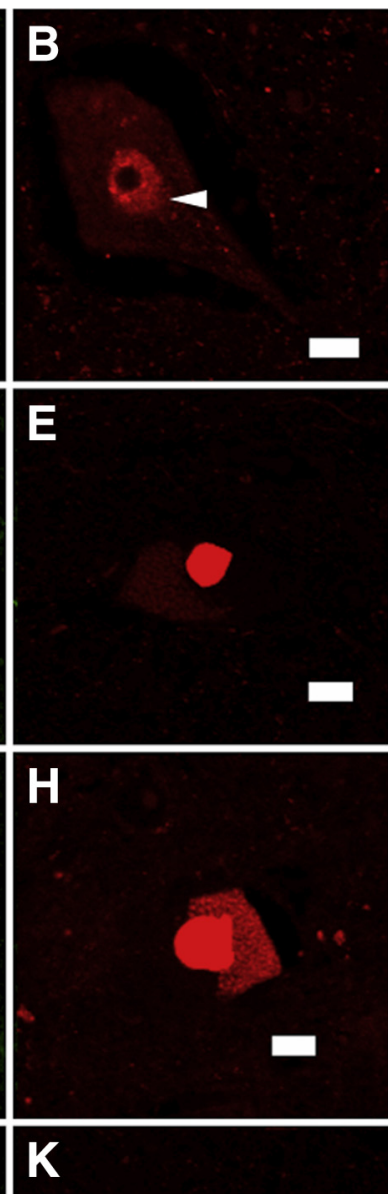

and TDP-43 in a subset of NCIs in motor neurons was confirmed (Figure 3). The co-localization of MATR3 and pTDP-43 was also confirmed (Supplemental Figure S5). Because no MATR3-positive and pTDP-43-negative NCIs were observed, double-positive NCIs were estimated as $16.8 \%$ ( $n=18 / 107$ ) (Table 3 ). Neurons without TDP-43and MATR3-positive NCIs often exhibited the strong nuclear staining pattern (Figure 3, A-C), whereas those with TDP-43- and MATR3-positive NCIs exhibited mild nuclear staining pattern for MATR3 (Table 3 and Figure 3, D-I). Interestingly, neurons with TDP-43-positive but MATR3-negative NCIs also exhibited mild nuclear staining pattern for MATR3, similar to the pattern of TDP-43 (Figure 3, J-L). Although most TDP-43-positive NCIs did not exhibit MATR3 immunoreactivity, all MATR3positive NCIs were TDP-43 positive.
Figure 3 Immunofluorescence staining for matrin 3 (MATR3) and transactivating response region DNA-binding protein 43 (TDP-43). MATR3 and TDP-43 are visualized with Alexa Fluor 488 (green) and Alexa Fluor 568 (red), respectively. A-C: Motor neuron without MATR3-positive (A) and TDP-43-positive (B) neuronal cytoplasmic inclusions (NCIs). D-I: Motor neurons with MATR3-positive (D and G) and TDP-43positive (E and $\mathbf{H}$ ) round NCIs. J-L: Motor neuron with TDP-43-positive (K) but MATR3-negative (J) skein-shaped and round NCIs. Merged images for each panel are shown in C, F, I, and L. Nuclei are indicated by arrowheads. Scale bars $=10 \mu \mathrm{m}$.

\section{Discussion}

In this study, the involvement of MATR3 in SALS pathology was demonstrated using 15 SALS and seven control autopsied cases. Previously, only one study has demonstrated the pathologic involvement of MATR3 in motor neurons of SALS, although our results partly differ from published findings. ${ }^{15}$

First, MATR3-positive NCIs were detected in more than half of SALS cases, whereas the previous report detected MATR3-positive NCIs in only one case that harbored C9orf72 hexanucleotide repeat expansion. ${ }^{15}$ By contrast, the nine SALS cases with MATR3-positive NCIs were pathologically distinct from C9orf72-related ALS, specifically in terms of the absence of ubiquitin-positive and TDP-43-negative cytoplasmic inclusions in the cerebellum 
and hippocampus. Moreover, six ALS cases, including three cases with MATR3-positive NCIs, were negative for the GGGGCC hexanucleotide expansion. Considering that C9orf72-related ALS cases are extremely rare in the Japanese population, ${ }^{22}$ it is unlikely that any of these cases harbored a $C 9$ orf 72 hexanucleotide repeat expansion. These results indicated the broader involvement of MATR3 in the pathomechanisms of SALS. In addition, four types of commercially available anti-MATR3 antibodies were validated, and a single antibody (A300-590A), which was proven to detect NCIs, was selected for subsequent evaluations. Differences in the antibodies used might explain the discrepancies between these findings and the previous report. ${ }^{15}$

Second, MATR3 co-localized with a subset of TDP43-positive NCIs. A previous report presumed that MATR3 interacts with TDP-43. ${ }^{23,24}$ Nevertheless, co-localization of MATR3 and TDP-43 has not been previously reported. This result strongly suggests that MATR3 is associated with TDP-43 pathology in SALS. In addition, a smaller number of MATR3-positive NCIs than TDP-43-positive NCIs and a lack of MATR3-positive and TDP-43-negative NCIs indicated that MATR3 interaction with NCIs is likely to occur downstream of formation of TDP-43-positive NCIs.

Third, strong MATR3 staining patterns in nuclei of motor neurons or glial cells were not specific pathologic findings of SALS because similar staining patterns were also observed in control cases. Consistent with our findings, a previous immunohistochemical study reported both strong and mild nuclear staining patterns of MATR3 in neurons of wild-type mouse. ${ }^{25}$ In SALS cases, most motor neurons with MATR3positive NCIs exhibited mild nuclear staining, whereas more than half of the remaining motor neurons without NCIs exhibited strong nuclear staining (Table 3 and Figures 1 and 3 ). This result indicated the possibility of translocation of MATR3 from the nucleus to the cytoplasm, in line with the progression of TDP-43 pathology. A recent report found that overexpression of a mutant MATR3 associated with ALS and distal myopathy does not induce cytoplasmic or nuclear inclusion structures in cultured cell lines. ${ }^{26}$ In addition, the autopsied cases that harbored a MATR3 mutation (p.Phe115Cys) did not exhibit NCIs. On the basis of these findings, translocation of MATR3 from the nucleus to NCIs seems specific to SALS.

The cases with MATR3-positive NCIs had significantly shorter disease duration (Table 4), implying a rapid progressive course. The larger number of remaining anterior horn cells in cases with shorter disease duration might increase the feasibility of detecting MATR3-positive NCIs. Otherwise, the presence of MATR3 in NCIs might increase neuronal toxicity and accelerate neuronal dysfunction.

MATR3 is an RNA- and DNA-binding nuclear matrix protein. ${ }^{10}$ Although MATR3 is associated with FALS $^{15}$ and vocal cord and pharyngeal weakness with distal myopathy, ${ }^{13,14}$ its function remains largely unknown; however, it has been suspected to be relevant to RNA metabolism on the basis of its RNA-binding domains and interactions with other RNA-binding proteins. ${ }^{27}$ Using a combination of RNA interference knockdown, transcriptome profiling, and iCLIP, Coelho et $\mathrm{al}^{28}$ found that MATR3 is a strong regulator of multiple splicing events. MATR3 has also been reported to be involved in the DNA damage response. ${ }^{29}$ Our results suggest that dysfunction of MATR3 might occur in motor neurons with MATR3-positive NCIs through translocation from the nucleus, where it primarily exerts DNA/RNA metabolism. We speculate that dysfunction of MATR3 at least partly contributes to the molecular pathogenesis in SALS, as well as FALS with MATR3 mutation.

This novel observation of MATR3 involvement in SALS pathogenesis in this cohort reveals a new key factor in a common pathway of disordered RNA metabolism that includes TDP-43, FUS/TLS, and many other components of NCIs. ${ }^{30}$ Further functional studies are needed to unveil the role of MATR3 aggregation in SALS pathology.

\section{Acknowledgment}

We thank the Japanese Consortium for Amyotrophic Lateral Sclerosis Research for providing the DNA samples from patients with the $C 9$ orf 72 hexanucleotide repeat expansion as controls for genetic analyses.

\section{Supplemental Data}

Supplemental material for this article can be found at https://doi.org/10.1016/j.ajpath.2017.10.007.

\section{References}

1. Arai T, Hasegawa M, Akiyama H, Ikeda K, Nonaka T, Mori H, Mann D, Tsuchiya K, Yoshida M, Hashizume Y, Oda T: TDP-43 is a component of ubiquitin-positive tau-negative inclusions in frontotemporal lobar degeneration and amyotrophic lateral sclerosis. Biochem Biophys Res Commun 2006, 351:602-611

2. Neumann M, Sampathu DM, Kwong LK, Truax AC, Micsenyi MC, Chou TT, Bruce J, Schuck T, Grossman M, Clark CM, McCluskey LF Miller BL, Masliah E, Mackenzie IR, Feldman H, Feiden W, Kretzschmar HA, Trojanowski JQ, Lee VM: Ubiquitinated TDP-43 in frontotemporal lobar degeneration and amyotrophic lateral sclerosis. Science 2006, 314:130-133

3. Kabashi E, Valdmanis PN, Dion P, Spiegelman D, McConkey BJ, Vande Velde C, Bouchard JP, Lacomblez L, Pochigaeva K, Salachas F, Pradat PF, Camu W, Meininger V, Dupre N, Rouleau GA: TARDBP mutations in individuals with sporadic and familial amyotrophic lateral sclerosis. Nat Genet 2008, 40:572-574

4. Sreedharan J, Blair IP, Tripathi VB, Hu X, Vance C, Rogelj B, Ackerley S, Durnall JC, Williams KL, Buratti E, Baralle F, de Belleroche J, Mitchell JD, Leigh PN, Al-Chalabi A, Miller CC, Nicholson G, Shaw CE: TDP-43 mutations in familial and sporadic amyotrophic lateral sclerosis. Science 2008, 21:1668-1672

5. DeJesus-Hernandez M, Mackenzie IR, Boeve BF, Boxer AL, Baker M, Rutherford NJ, Nicholson AM, Finch NA, Flynn H, Adamson J, Kouri N, Wojtas A, Sengdy P, Hsiung GY, Karydas A, Seeley WW, Josephs KA, Coppola G, Geschwind DH, Wszolek ZK, Feldman H, Knopman DS, Petersen RC, Miller BL, Dickson DW, Boylan KB, 
Graff-Radford NR, Rademakers R: Expanded GGGGCC hexanucleotide repeat in noncoding region of C9ORF72 causes chromosome 9plinked FTD and ALS. Neuron 2011, 72:245-256

6. Deng HX, Zhai H, Bigio EH, Yan J, Fecto F, Ajroud K, Mishra M, Ajroud-Driss S, Heller S, Sufit R, Siddique N, Mugnaini E, Siddique T: FUS-immunoreactive inclusions are a common feature in sporadic and non-SOD1 familial amyotrophic lateral sclerosis. Ann Neurol 2010, 67:739-748

7. Vance C, Rogelj B, Hortobágyi T, De Vos KJ, Nishimura AL, Sreedharan J, Hu X, Smith B, Ruddy D, Wright P, Ganesalingam J, Williams KL, Tripathi V, Al-Saraj S, Al-Chalabi A, Leigh PN, Blair IP, Nicholson G, de Belleroche J, Gallo JM, Miller CC, Shaw CE: Mutations in FUS, an RNA processing protein, cause familial amyotrophic lateral sclerosis type 6. Science 2009, 323:1208-1211

8. Deng HX, Chen W, Hong ST, Boycott KM, Gorrie GH, Siddique N, Yang Y, Fecto F, Shi Y, Zhai H, Jiang H, Hirano M, Rampersaud E, Jansen GH, Donkervoort S, Bigio EH, Brooks BR, Ajroud K, Sufit RL, Haines JL, Mugnaini E, Pericak-Vance MA, Siddique T: Mutations in UBQLN2 cause dominant X-linked juvenile and adult-onset ALS and ALS/dementia. Nature 2011, 477:211-215

9. Da Cruz S, Bui A, Saberi S, Lee SK, Stauffer J, McAlonis-Downes M, Schulte D, Pizzo DP, Parone PA, Cleveland DW, Ravits J: Misfolded SOD1 is not a primary component of sporadic ALS. Acta Neuropathol 2017, 134:97-111

10. Belgrader P, Dey R, Berezney R: Molecular cloning of matrin 3. A 125- kilodalton protein of the nuclear matrix contains an extensive acidic domain. J Biol Chem 1991, 266:9893-9899

11. Nakayasu H, Berezney R: Nuclear matrins: identification of the major nuclear matrix proteins. Proc Natl Acad Sci U S A 1991, 88:10312-10316

12. Hisada-Ishii S, Ebihara M, Kobayashi N, Kitagawa Y: Bipartite nuclear localization signal of matrin 3 is essential for vertebrate cells. Biochem Biophys Res Commun 2007, 354:72-76

13. Senderek J, Garvey SM, Krieger M, Guergueltcheva V, Urtizberea A, Roos A, Elbracht M, Stendel C, Tournev I, Mihailova V, Feit H, Tramonte J, Hedera P, Crooks K, Bergmann C, Rudnik-Schoneborn S, Zerres K, Lochmuller H, Seboun E, Weis J, Beckmann JS, Hauser MA, Jackson CE: Autosomal-dominant distal myopathy associated with a recurrent missense mutation in the gene encoding the nuclear matrix protein, matrin 3. Am J Hum Genet 2009, 84:511-518

14. Muller TJ, Kraya T, Stoltenburg-Didinger G, Hanisch F, Kornhuber M, Stoevesandt D, Senderek J, Weis J, Baum P, Deschauer M, Zierz S: Phenotype of matrin-3-related distal myopathy in 16 German patients. Ann Neurol 2014, 76:669-680

15. Johnson JO, Pioro EP, Boehringer A, Chia R, Feit H, Renton AE, Pliner HA, Abramzon Y, Marangi G, Winborn BJ, Gibbs JR, Nalls MA, Morgan S, Shoai M, Hardy J, Pittman A, Orrell RW, Malaspina A, Sidle KC, Fratta P, Harms MB, Baloh RH, Pestronk A, Weihl CC, Rogaeva E, Zinman L, Drory VE, Borghero G, Mora G, Calvo A, Rothstein JD; ITALSGEN, Drepper C, Sendtner M, Singleton AB, Taylor JP, Cookson MR, Restagno G, Sabatelli M, Bowser R, Chio A, Traynor BJ: Mutations in the Matrin 3 gene cause familial amyotrophic lateral sclerosis. Nat Neurosci 2014, 17:664-666

16. Lin KP, Tsai PC, Liao YC, Chen WT, Tsai CP, Soong BW, Lee YC: Mutational analysis of MATR3 in Taiwanese patients with amyotrophic lateral sclerosis. Neurobiol Aging 2015, 36:2005.e1-2005.e4

17. Marangi G, Lattante S, Doronzio PN, Conte A, Tasca G, Monforte M, Patanella AK, Bisogni G, Meleo E, La Spada S, Zollino M, Sabatelli M:
Matrin 3 variants are frequent in Italian ALS patients. Neurobiol Aging 2017, 49:218.e1-218.e7

18. Brettsschneider J, Tredici KD, Toledo JB, Robinson JL, Irwin DJ, Grossman M, Suh E, Van Deerlin VM, Wood EM, Baek Y, Kwong L, Lee E, Elman L, McCluskey L, Fang L, Feldengut S, Ludolph AC, Lee VM, Braak H, Trojanowski JQ: Stages of pDP-43 pathology in amyotrophic lateral sclerosis. Ann Neurol 2013, 74:20-38

19. Al-Sarraj S, King A, Troakes C, Smith B, Maekawa S, Bodi I, Rogelj B, Al- Chalabi A, Hortobagyi T, Shaw CE: P62 positive, TDP-43 negative, neuronal cytoplasmic and intranuclear inclusions in the cerebellum and hippocampus define the pathology of C9orf72-linked FTLD and MND/ALS. Acta Neuropathol 2011, 122: 691-702

20. Mackenzie IR, Arzberger T, Kremmer E, Troost D, Lorenzl S, Mori K, Weng SM, Haass C, Kretzschmar HA, Edbauer D, Neumann M: Dipeptide repeat protein pathology in C9ORF72 mutation cases: clinico-pathological correlations. Acta Neuropathol 2013, 126: 859-879

21. Mori K, Weng SM, Arzberger T, May S, Rentzsch K, Kremmer E, Schmid B, Kretzschmar HA, Cruts M, Van Broeckhoven C, Haass C, Edbauer D: The C9orf72 GGGGCC repeat is translated into aggregating dipeptide-repeat proteins in FTLD/ALS. Science 2013, 339: $1335-1338$

22. Ogaki K, Li Y, Atsuta N, Tomiyama H, Funayama M, Watanabe H, Nakamura R, Yoshino H, Yato S, Tamura A, Naito Y, Taniguchi A, Fujita K, Izumi Y, Kaji R, Hattori N, Sobue G; Japanese Consortium for Amyotrophic Lateral Sclerosis (JaCALs): Analysis of C9orf72 repeat expansion in 563 Japanese patients with amyotrophic lateral sclerosis. Neurobiol Aging 2012, 33:2527.e11-2527.e16

23. Ling SC, Albuquerque CP, Han JS, Lagier-Tourenne C, Tokunaga S, Zhou H, Cleveland DW: ALS-associated mutations in TDP-43 increase its stability and promote TDP-43 complexes with FUS/TLS. Proc Natl Acad Sci U S A 2010, 107:13318-13323

24. Salton M, Elkon R, Borodina T, Davydov A, Yaspo ML, Halperin E, Shiloh Y: Matrin 3 binds and stabilizes mRNA. PLoS One 2011, 6 : e23882

25. Rayaprolu S, D’Alton S, Crosby K, Moloney C, Howard J, Duffy C, Cabrera M, Siemienski Z, Hernandez AR, Gallego-Iradi C, Borchelt DR, Lewis J: Heterogeneity of Matrin 3 in the developing and aging murine central nervous system. J Comp Neurol 2016, 524: $2740-2752$

26. Gallego-Iradi MC, Clare AM, Brown HH, Janus C, Lewis J, Borchelt DR: Subcellular localization of Matrin 3 containing mutations associated with ALS and distal myopathy. PLoS One 2015, 10: $\mathrm{e} 0142144$

27. Zeitz MJ, Malyavantham KS, Seifert B, Berezney R: Matrin 3: chromosomal distribution and protein interactions. J Cell Biochem 2009, 108:125-133

28. Coelho MB, Attig J, Bellora N, Konig J, Hallegger M, Kayikci M, Eyras E, Ule J, Smith CW: Nuclear matrix protein Matrin3 regulates alternative splicing and forms overlapping regulatory networks with PTB. EMBO J 2015, 34:653-668

29. Salton M, Lerenthal Y, Wang SY, Chen DJ, Shiloh Y: Involvement of Matrin 3 and SFPQ/NONO in the DNA damage response. Cell Cycle 2010, 9:1568-1576

30. Strong MJ: The evidence for altered RNA metabolism in amyotrophic lateral sclerosis(ALS). J Neurol Sci 2010, 288:1-12 